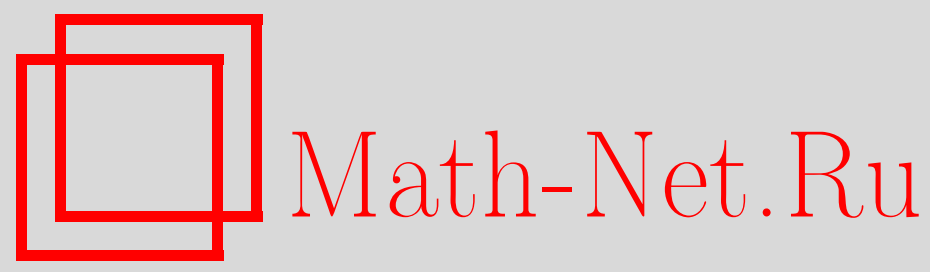

В. С. Климов, Теоремы об устойчивости по первому приближению для дифференциальных включений, Матем. заметки, 2004, том 76, выпуск 4, 517-530

DOI: https://doi.org/10.4213/mzm128

Использование Общероссийского математического портала Math-Net.Ru подразумевает, что вы прочитали и согласны с пользовательским соглашением http://www.mathnet.ru/rus/agreement

Параметры загрузки:

IP: 18.207 .199 .55

26 апреля 2023 г., 14:16:48

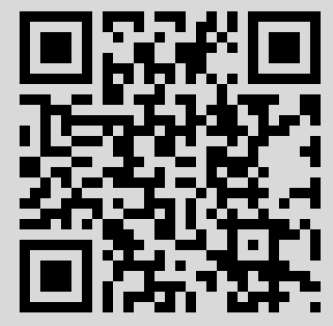




\title{
ТЕОРЕМЫ ОБ УСТОЙЧИВОСТИ ПО ПЕРВОМУ ПРИБЛИЖЕНИЮ ДЛЯ ДИФФЕРЕНЦИАЛЬНЫХ ВКЛЮЧЕНИЙ
}

\section{В. С. Климов}

\begin{abstract}
Устанавливаются многозначные и бесконечномерные варианты теорем об устойчивости по первому приближению. Дифференциальные включения, рассматриваемые в качестве первого приближения, могут быть неавтономными, а в ряде изучаемых случаев и неоднородными по фазовому переменному. Намечены приложения к теории устойчивости решений параболических включений.

Библиограффия: 16 названий.
\end{abstract}

Ниже $\|x ; E\|$ - норма элемента $x$ в $B$-пространстве $E, E^{*}-$ сопряженное к $E$ пространство, $\left(x, x^{*}\right)$ - значение функционала $x^{*}$ из $E^{*}$ на элементе $x \in E, \sigma\left(E, E^{*}\right)$ и $\sigma\left(E^{*}, E\right)$ - слабые топологии на $E$ и $E^{*}$ соответственно, порождаемые формой $(\cdot, \cdot)$;

$$
s\left(x^{*}, \mathscr{D}\right)=\sup \left\{\left(x, x^{*}\right), x \in \mathscr{D}\right\}, \quad x^{*} \subset E^{*},
$$

- опорная функция множества $\mathscr{D} \subset E$,

$$
\theta_{E}\left(\mathscr{D}_{1}, \mathscr{D}_{2}\right)=\sup _{x_{1} \in \mathscr{D}_{1}} \inf _{x_{2} \in \mathscr{D}_{2}}\left\|x_{1}-x_{2} ; E\right\|
$$

- уклонение множества $\mathscr{D}_{1} \subset E$ от множества $\mathscr{D}_{2} \subset E ; L^{p}(J, E)($ соответственно $C(J, E))$ - пространство измеримых по Лебегу-Бохнеру (соответственно непрерьвных) на отрезке $J \subset \mathbb{R}$ функций со значениями в $E$, как обычно, эквивалентные функции отождествляются, нормы в $C(J, E)$ и $L^{p}(J, E)$ определяются стандартным образом (см., например, $[1$, гл. $4, \S \mathrm{I}]) ; L_{w}^{p}(j, E)$ - топологическое пространство слабо измеримых на отрезке $J$ функций со значениями в $E$, определяющая в $L_{w}^{p}(J, E)$ топологию система полунорм задается равенством

$$
\|v\|=\left\|l(v) ; L^{p}(J)\right\|, \quad \text { где } l \in E^{*}, \quad L^{p}(J)=L^{p}(J, \mathbb{R}) ;
$$

все банаховыпространства рассматриваются над полем $\mathbb{R}$ действительных чисел; $\operatorname{Cv}(E)$ означает совокупность непустых замкнутых и вьпуклых подмножеств $E ; E_{1} \times E_{2}-$ прямое произведение $B$-пространств $E_{1}, E_{2}$; если $E_{1}, E_{2}$ непрерывно вложены в локально выпуклое топологическоепространство $E_{0}$, то с $E_{1}, E_{2}$ связаны $B$-пространства $E_{1} \cap E_{2}$ и $E_{1}+E_{2}$ (см. [2, гл. $\left.\left.1, \S 5\right]\right)$; запись $E_{1} \rightarrow E_{2}$ (соответственно $E_{1} \stackrel{\mathrm{k}}{\rightarrow} E_{2}$ ) означает, что $E_{1}$ непрерывно (соответственно компактно) вложено в $E_{2}$. 
Многозначное отображение (m-отображение) $F$ множества $\mathscr{D}_{1}$ в множество $\mathscr{D}_{2}-$ это оператор, сопоставляющий каждой точке $v$ из $\mathscr{D}_{1}$ непустое множество $F(v) \subset \mathscr{D}_{2}$, назьваемое образом точки $v$; если $\mathscr{D} \subset \mathscr{D}_{1}$, то $F(\mathscr{D})=U F(v), v \in \mathscr{D},-$ область значений отображения $F$ на множестве $\mathscr{D}$. $M$-отображение $F: \mathscr{D}_{1} \rightarrow \mathscr{D}_{2}$ (где $\mathscr{D}_{1}, \mathscr{D}_{2}$ - подмножества $B$-пространств $\left.E_{1}, E_{2}\right)$ называют хеминепрерывным сверху на $\mathscr{D}_{1}$ (см. [2, гл. 3 , п. 2]), если для каждого $x^{*}$ из $E_{2}^{*}$ функция $v \rightarrow s\left(x^{*}, F(v)\right)$ полунепрерьвна сверху на $\mathscr{D}_{1}$. Если образы $m$-отображения $F: \mathscr{D} \rightarrow E$ принадлежат классу $\mathrm{Cv}(E)$, то будем записывать следующим образом: $F: \mathscr{D} \rightarrow \mathrm{Cv}(E)$.

1. Постановка задачи. Пусть $V$-рефлексивное сепарабельное $B$-пространство с нормой $\|\cdot\|, V^{*}$ - сопряженное к нему пространство с нормой $\|\cdot\|_{*}, H$ - гильбертово пространство с нормой $|\cdot|, V \stackrel{\mathrm{k}}{\rightarrow} H$ и $V$ плотно в $H$. Пространство $H$ отождествляется с сопряженным к нему $H^{*}$, а $H^{*}=H-$ с подпространством пространства $V^{*}$. Число $\left(v, v^{*}\right)$ означает и скалярное произведение элементов $v, v^{*}$ из $H$, и значение функционала $v^{*}$ из $V^{*}$ на элементе $v \in V$.

Сопоставим отрезку $J \subset \mathbb{R}$ банаховы пространства

$$
Y=L^{2}(J, V) \cap L^{\infty}(J, H) \quad \text { и } \quad Z=L^{2}\left(J, V^{*}\right)+L^{1}(J, H) .
$$

Пространство $Y$ можно идентифицировать с сопряженньм к пространству $Z$ (см. [1, гл. $4, \S 1])$. Билинейная форма, определяющая двойственность, задается равенством $\langle y, z\rangle=\int(y(t), z(t)) d t$. Если необходимо подчеркнуть зависимость пространств $Y, Z$ от $J$, будем обозначать их символами $Y(J), Z(J)$.

Положим

$$
W=\left\{y \mid y \in Y, y^{\prime} \in \mathbb{Z}\right\} .
$$

Равенство $y^{\prime}+z=0$ означает, что

$$
\left\langle y, \varphi^{\prime} v\right\rangle=\langle\varphi v, z\rangle, \quad \varphi \in C_{0}^{\infty}(J), \quad v \in V
$$

Пространство $W$ вложено в $C(J, H)$; это позволяет ввести понятие значения функции из $W$ в каждой точке отрезка $J$. Если $y \in W$, то функция $|y(t)|^{2}$ абсолютно непрерьвна на $J$ и справедлива формула [1], [3]

$$
\left(|y(t)|^{2}\right)^{\prime}=2\left(y(t), y^{\prime}(t)\right) \quad \text { п.в. }
$$

Через $Y_{0}$ обозначим линейное пространство $Y$, наделенное слабейшей из топологий, мажорируюшей топологии $\sigma(Y, Z)$ и $L_{w}^{2}(J, H)$. Пространство $Y_{0}$ непрерывно вложено в $L^{2}(J, H)$ (см. [4]).

Введем специальный класс $m$-отображений на пространстве $Y=Y(J), J=\left[t_{0}, t_{1}\right]$. Обозначим через $\alpha_{1}(Y, Z)$ совокупность операторов $A: Y \rightarrow \operatorname{Cv}(Z)$, удовлетворяюших условиям

1) оператор $A$ усиленно ограничен в том смысле, что для каждого ограниченного множества $\mathscr{D} \subset Y$ множество $A(\mathscr{D})$ предкомпактно в топологии $\sigma(Z, Y)$;

2) для произвольных последовательностей $y_{i} \in Y, z_{i} \in A\left(y_{i}\right)$, обладающих свойствами

$$
y_{i} \rightarrow y \quad \text { в } Y_{0}, \quad z_{i} \rightarrow z \quad \text { в } \quad \sigma(Z, Y), \quad \varlimsup_{i \rightarrow \infty}\left\langle y_{i}, z_{i}\right\rangle \leqslant\langle y, z\rangle
$$


имеет место включение $z \in A(y)$ и сходимость

$$
\left\langle 1_{\left[t_{0}, t\right]} y_{i}, z_{i}\right\rangle \rightarrow\left\langle 1_{\left[t_{0}, t\right]} y, z\right\rangle
$$

в $C(J)$ (здесь $1_{\left[t_{0}, t\right]}$ - характеристическая функция отрезка $\left.\left[t_{0}, t\right] \subset J\right)$.

Второе условие созвучно требованию усиленной замкнутости [5] оператора $A$.

Многозначньй оператор $A: Y \rightarrow Z$ назовем локальныцм, если существует семейство операторов $\mathscr{A}(t): V \rightarrow V^{*}(t \in J)$, порождающее $A$ в том смысле, что для любого $y$ из $Y$ множество $A(y)$ совпадает с множеством измеримых сечений $m$-отображения $t \rightarrow \mathscr{A}(t, y(t))$ (здесь и далее $\mathscr{A}(t, v)=\mathscr{A}(t) v)$. Примеры локальных операторов класса $\alpha_{1}(Y, Z)$ приведены в [4], [6].

Пусть $A$ - локальньй оператор класса $\alpha_{1}(Y, Z)$, порождаемый семейством отображений

$$
\mathscr{A}(t): V \rightarrow V^{*}, \quad t \in J=\left[t_{0}, t_{1}\right] .
$$

Рассмотрим дифференциальное включение

$$
0 \in y^{\prime}+\mathscr{A}(t, y)
$$

Под его решением будем понимать функцию $y$ класса $W$, для которой $-y^{\prime} \in A(y)$. Задача Коши для включения (1) состоит в отыскании решения $y$, удовлетворяющего начальному условию

$$
y\left(t_{0}\right)=h, \quad h \in H
$$

Ниже

$$
\operatorname{Gr} A=\{(y, z) \in Y \times Z, y \in Y, z \in A(y)\}
$$

- график отображения $A: Y \rightarrow \mathrm{Cv}(Z)$,

$$
\theta_{Y \times Z}((x, g) ; \operatorname{Gr} A)=\inf \{\|x-y ; Y\|+\|g-z ; Z\|, y \in Y, z \in A(y)\}
$$

- расстояние в метрике $Y \times Z$ от $(x, g)$ до графика отображения $A$.

ПРЕДЛОЖЕНИЕ 1. Пусть $x_{n} \in W, g_{n}=-x_{n}^{\prime}, x_{n} \rightarrow x$ в $\sigma(Y, Z)$,

$$
\theta_{Y \times Z}\left(\left(x_{n}, g_{n}\right) ; \operatorname{Gr} A\right) \rightarrow 0 \quad n p u \quad n \rightarrow \infty .
$$

\section{Тогда}

1) $x$ есть решение включения (1) $u\left(t-t_{0}\right) x_{n} \rightarrow\left(t-t_{0}\right) x$ в $C(J, H)$;

2) если $x_{n}\left(t_{0}\right) \rightarrow$ в в метрике $H$, то $x$ есть решение задачи Коши (1), (2) и $x_{n} \rightarrow x$ \& $C(J, H)$. 
Предложение 1 есть вариант леммы 1 работы [7].

Далее включение (1) изучается в случае, когда $t$ изменяется на луче $\mathbb{R}_{+}=[0, \infty)$. Введем пространство $M_{p}\left(\mathbb{R}_{+}, E\right)$, ограниченных по Степанову функций на $\mathbb{R}_{+}$со значениями в $B$-пространстве $E$. Оно состоит из измеримых по Лебегу-Бохнеру функций $f: \mathbb{R}_{+} \rightarrow E$, для которьх имеет смысл и конечна норма

$$
\left\|f ; M_{p}\right\|=\sup \left\{\left\|f ; L^{p}(I, E)\right\|\right\}
$$

где sup берется по всем отрезкам $I \subset \mathbb{R}_{+}$единичной длины. Полагаем $M_{p}\left(\mathbb{R}_{+}, \mathbb{R}\right)=$ $M_{p}\left(\mathbb{R}_{+}\right)$.

Будем писать $\mathscr{A} \in \mathbb{C}_{*}\left(\mathbb{R}_{+}, V\right)$, если операторы $\mathscr{A}(t): V \rightarrow V^{*}, t \in \mathbb{R}_{+}$, допускают представление

$$
\mathscr{A}(t)=\mathscr{A}_{1}(t)+\mathscr{A}_{2}(t)
$$

где $\mathscr{A}_{1}(t), \mathscr{A}_{2}(t)$ удовлетворяют условиям

$\left.c_{1}\right)$ почти при всех $t$ отображения $\mathscr{A}_{1}(t): V \rightarrow \mathrm{Cv}\left(V^{*}\right), \mathscr{A}_{2}(t): V \rightarrow \mathrm{Cv}(H)$ хеминепрерывны сверху;

$\left.c_{2}\right)$ существуют однозначные отображения $a_{i}(t, v) \subset A_{i}(t, v), i=1,2$, измеримые по $t$ при каждом $v$ из $V$;

$c_{3}$ ) для каждого $R>0$ справедливы неравенства

$$
\left\|v_{1}^{*}\right\|_{*} \leqslant k\|v\|+\gamma_{1}(t), \quad\left|v_{2}^{*}\right| \leqslant \gamma_{2}(t)
$$

в которых $|v| \leqslant R, v_{i}^{*} \in \mathscr{A}_{i}(t \mid v), i=1,2 ;$ константа $k>0$ и функции $\gamma_{1} \in M_{2}\left(\mathbb{R}_{+}\right)$, $\gamma_{2} \in M_{1}\left(\mathbb{R}_{+}\right)$могут зависеть от $R$.

Предположение $\mathscr{A} \in \mathbb{C}_{*}\left(\mathbb{R}_{+}, V\right)$ влечет суперпозиционную измеримость отображения $\mathscr{A}$ : множество $A(y)$ измеримых сечений отображения $t \rightarrow \mathscr{A}(t, y(t))$ непусто для каждого измеримого отображения $y: \mathbb{R}_{+} \rightarrow V$; сужение $A$ на пространство $Y(J)$, где $J=\left[t_{0}, t_{1}\right] \subset \mathbb{R}_{+}$, есть отображение со значениями в $\operatorname{Cv}(Z(J)) ;$ условие с 3$)$ влечет усиленную ограниченность оператора $A[4]$. В случае конечномерного пространства $V$ отображение $A$ принадлежит классу $\alpha_{1}(Y(J), Z(J))$ для любого отрезка $J \subset \mathbb{R}_{+} \cdot \mathrm{B}$ бесконечномерном пространстве $V$ аналогичный факт верен при определенных дополнительных предположениях. Полезным оказьвается следующее

УСловиЕ I. Существует такая неотрищательная функция $\lambda$ класса $M_{1}\left(\mathbb{R}_{+}\right)$, что

$$
\left(v_{1}-v_{2}, v_{1}^{*}-v_{2}^{*}\right) \geqslant-\lambda(t)\left|v_{1}-v_{2}\right|^{2}, \quad v_{j} \in V, \quad v_{j}^{*} \in \mathscr{A}\left(t, v_{j}\right), \quad j=1,2 .
$$

Неравенство (3) есть одностороннее условие Липшица для $m$-отображения $\mathscr{A}(t): V \rightarrow$ $V^{*}$. Оно заведомо вьполняется в случае монотонного отображения $\mathscr{A}$.

ПРЕДЛОЖЕНИЕ 2 [4]. Пусть $\mathscr{A} \in \mathbb{C}_{*}\left(\mathbb{R}_{+}, V\right)$ и выполнено условие I. Тогда порождаемый $\mathscr{A}$ оператор суперпозичии $A$ принадлежст классу $\alpha_{1}(Y(J), Z(J))$ для любого отрезка $J \subset \mathbb{R}_{+}$.

Более широкие условия на $m$-отображение $\mathscr{A}: \mathbb{R}_{+} \times V \rightarrow V^{*}$, гарантирующие включение $A \in \alpha_{1}(Y(J), Z(J))$, можно найти в [4], [6]. Близкое к условию I условие полуограниченности вариации однозначного отображения широко применяется в [8]. 
Если $0 \in \mathscr{A}(t, 0), t \in \mathbb{R}_{+}$, то функция $y_{0}(t) \equiv 0$ есть решение включения (1). Назовем нулевое решение асимптотически устойчивым, если

1) для каждого $\varepsilon>0$ существует такое $\delta>0$, что любое решение $x(t)$ включения (1) с $|x(0)|<\delta$ существует при $t \geqslant 0$ и удовлетворяет неравенству $|x(t)|<\varepsilon$, $t \in \mathbb{R}_{+} ;$

2) $|x(t)| \rightarrow 0$ при $t \rightarrow \infty$.

Нулевое решение назовем әкспоненциально устойчивым в малом, если можно указать такое $\varepsilon>0$, что любое решение $x(t)$ включения (1) при $\left|x\left(t_{0}\right)\right|<\varepsilon, t \geqslant t_{0}$ удовлетворяет неравенству

$$
|x(t)| \leqslant B\left|x\left(t_{0}\right)\right| e^{-\alpha\left(t-t_{0}\right)},
$$

где постоянные $\alpha>0, B \geqslant 1$ не зависят от $t_{0}$.

Приведенные определения близки к принятым в [9], [10]. Известны и другие определения устойчивости тривиального решения, вообе говоря, не эквивалентные приведенньм выше.

Введем в рассмотрение семейство $m$-отображений

$$
\mathscr{A}_{\rho}: \mathbb{R}_{+} \times V \rightarrow V^{*}
$$

полагая

$$
\mathscr{A}_{\rho}(t, v)=\mathscr{A}(t, v)+\rho\|v\| \mathbb{B}_{V^{*}},
$$

где $\rho \geqslant 0, t \geqslant 0, v \in V, \mathbb{B}_{V^{*}}=\left\{v^{*} \in V^{*},\left\|v^{*}\right\|_{*} \leqslant 1\right\}$. M-отображение $\mathscr{A}_{\rho}$ при любом $\rho \geqslant 0$ принадлежит классу $\mathbb{C}_{*}\left(\mathbb{R}_{+}, V\right)$. Если $0 \in \mathscr{A}(t, 0), t \geqslant 0$, то $0 \in \mathscr{A}_{\rho}(t, 0), t \geqslant 0$, $\rho \geqslant 0$, поэтому дифференциальное включение

$$
0 \in y^{\prime}+\mathscr{A}_{\rho}(t, y)
$$

имеет нулевое решение. Далее формулируются предположения относительно $m$-отображения $\mathscr{A}$, при которых устойчивость (в некотором смысле) тривиального решения включения (1) влечет экспоненциальную устойчивость в малом нулевого решения включения (5) (при $0 \leqslant \rho<\rho_{0}, \rho_{0}>0$ ). У тверждения такого рода назьвают теоремами об устойчивости по первому приближению; подобные теоремы хорошо известны для дифференциальных уравнений. Ниже соответствующие результаты переносятся на некоторые классы дифференциальных включений в $B$-пространстве.

2. Теоремы об устойчивости по первому приближению. В этом пункте

$$
\mathscr{A} \in \mathbb{C}_{*}\left(\mathbb{R}_{+}, V\right), \quad 0 \in \mathscr{A}(t, 0), \quad t \geqslant 0 .
$$

Будет использоваться следующий вариант условия эллиптичности.

УСловиЕ II. Существует функция $\gamma_{0}$ класса $M_{1}\left(\mathbb{R}_{+}\right)$и константа $k_{1}>0$ такие, что

$$
\left(v, v^{*}\right)+\gamma_{0}(t)|v|^{2} \geqslant k_{1}\|v\|^{2},
$$

где $v \in V, v^{*} \in \mathscr{A}(t, v), t \geqslant 0$. 
Если выполнено условие II и порождаемый $\mathscr{A}$ оператор суперпозиции $A$ принадлежит классу $\alpha_{1}(Y(J), Z(J))$ для любого отрезка $J \subset \mathbb{R}$, то задача Коши $(1),(2)$ имеет решение $x(t)$, определенное при всех $t \geqslant t_{0}[4]$. Единственность решения задачи Коши $(1),(2)$ при этом не гарантируется. Если $x(t)$ - решение включения $(1)$, то из (6) вытекает дифференциальное неравенство

$$
\frac{1}{2}\left(|x(t)|^{2}\right)^{\prime}+k_{1}\|x(t)\|^{2} \leqslant \gamma_{0}(t)|x(t)|^{2}
$$

стандартным образом приводящее к оценкам

$$
\begin{aligned}
& |x(t)| \leqslant\left|x\left(t_{0}\right)\right| \exp \int_{t_{0}}^{t} \gamma_{0}(\tau) d \tau, \\
& \int_{t_{0}}^{t_{0}+T}\|x(t)\|^{2} d t \leqslant k^{2}(T)\left|x\left(t_{0}\right)\right|^{2} .
\end{aligned}
$$

Полезно заметить, что оценка (7) сохраняется и в случае, когда (6) справедливо с $k_{1}=0$. Постоянная $k(T)>0$ в (8) зависит лишш от $T$ и постоянной $k_{1}>0$ в неравенстве (6).

При достаточно малом $\rho>0$ неравенство (6) сохраняется для отображений $\mathscr{A}_{\rho}(t)$. Именно, при $0 \leqslant \rho<k_{1}$ справедливо вытекающее из (6) неравенство $\left(v, v^{*}\right)+\gamma_{0}(t)|v|^{2} \geqslant$ $\left(k_{1}-\rho\right)\|v\|^{2}$, где $v \in V, v^{*} \in \mathscr{A}_{\rho}(t, v)$. Это неравенство аналогично (6), поэтому для решения $y(t)$ включения (5) имеют место оценки

$$
|y(t)| \leqslant\left|y\left(t_{0}\right)\right| \exp \int_{t_{0}}^{t} \gamma_{0}(\tau) d \tau, \quad \exp \int_{t_{0}}^{t_{0}+T}\|y(t)\|^{2} d \tau \leqslant \varkappa^{2}(T)\left|y\left(t_{0}\right)\right|^{2}
$$

постоянная $\varkappa(T)>0$ в (9) зависит лиш от $T$ и $k_{1}-\rho>0$.

Если $m$-отображение $\mathscr{A}$ в $(9)$ удовлетворяет одностороннему условию Липшица I, то решение задачи Коши (1), (2) единственно. В этом случае можно оценить разницу между решениями включений (1), (5), удовлетворяющих одинаковым начальньм условиям что позволяет получить условия устойчивости нулевого решения включения (5).

Теорема 1. Пусть т-отображсение $\mathscr{A}$ класса $\mathbb{C}_{*}\left(\mathbb{R}_{+}, V\right)$ удовлетворяет условиям I, II и нулевое решение включения (1) әкспоненииально устойчиво в малом. Тогда найдется такое $\rho_{0}>0$, что при $\rho \in\left(0, \rho_{0}\right)$ тривиальное решение включения (5) также будет әкспоненциально устойчивым в малом.

ДокАЗАТЕЛЬСТво. Пусть $0<\rho<k_{1}, y$ - решение включения $(5), x-$ решение включения $(1)$ и $x\left(t_{0}\right)=y\left(t_{0}\right)$ при некотором $t_{0} \geqslant 0$. Справедливы равенства $0=x^{\prime}+w$, $0=y^{\prime}+z+\xi$, где $w, z, \xi$ - измеримые функции, $w(t) \in \mathscr{A}(t, x(t)), z(t) \in \mathscr{A}(t, y(t))$ и $\|\xi(t)\|_{*} \leqslant \rho\|y(t)\|$ п.в. Из условия I получаем последовательно

$$
\begin{aligned}
\frac{1}{2}\left(|y-x|^{2}\right)^{\prime} & =(y-x, w-z-\xi) \leqslant \lambda|y-x|^{2}-(y-x, \xi) \\
& \leqslant \lambda|y-x|^{2}+\rho\|y-x\|\|y\| \leqslant \lambda|y-x|^{2}+\frac{\rho}{2}\left(\|y-x\|^{2}+\|y\|^{2}\right) .
\end{aligned}
$$


Установленное дифференциальное неравенство и условие $x\left(t_{0}\right)=y\left(t_{0}\right)$ приводят при $t_{0} \leqslant t \leqslant t_{0}+T$ к оценке

$$
\begin{aligned}
|y(t)-x(t)|^{2} & \leqslant \rho \int_{t_{0}}^{t} \exp \left(2 \int_{\tau}^{t} \lambda(s) d s\right)\left(\|y(\tau)-x(\tau)\|^{2}+\|y(\tau)\|^{2}\right) d \tau \\
& \leqslant \rho T \exp \left(2 \int_{t_{0}}^{t_{0}+T} \lambda(s) d s\right) \int_{t_{0}}^{t_{0}+T}\left(\|y(\tau)-x(\tau)\|^{2}+\|y(\tau)\|^{2}\right) d \tau .
\end{aligned}
$$

Объединяя последнее неравенство с (9), (11), приходим к оценке

$$
|y(t)-x(t)| \leqslant \sqrt{\rho} \mu(T)\left|x\left(t_{0}\right)\right|
$$

постоянная $\mu(\cdot)$ зависит от $T$ и $\left\|\lambda ; M_{1}\right\|$.

В предшествующих рассуждениях не использовалась экспоненциальная устойчивость в малом нулевого решения включения (1). Пусть теперь для решений $x(t)$ включения (1) справедливо неравенство (4), если только $\left|x\left(t_{0}\right)\right|<\varepsilon$. Положим $T=\ln (4 B / \alpha)$. Если только $0<\rho<k_{1}$ и $\sqrt{\rho} \mu(T)<1 / 4$, то для пары решений $x(t), y(t)$ включений $(1),(5)$ соответственно, совпадаюших при $t=t_{0}$, справедливо вытекающее из (10) неравенство

$$
|y(t)-x(t)| \leqslant \frac{1}{4}\left|x\left(t_{0}\right)\right|, \quad t_{0} \leqslant t \leqslant t_{0}+T,
$$

влекущее [9, с. 191-193] экспоненциальную устойчивость в малом нулевого решения включения (5). Теорема доказана.

Отметим новые моменты, отличающие теорему 1 от аналогичных результатов в [9]:

1) отображение $\mathscr{A}(t)$ многозначно и действует не в одном пространстве, а из пространства $V$ в сопряженное к нему пространство $V^{*}$;

2) предполагаемое в [9] условие Липшица заменено его односторонним вариантом. Проверка экспоненциальной устойчивости нулевого решения включения (1) существенно упрощается, если выполнено

УСловИЕ III. Имеет место равенство $\mathscr{A}(t, c v)=c \mathscr{A}(t, v), t \geqslant 0, v \in V, c>0$.

Условие III означает 1-однородность $m$-отображения $\mathscr{A}$ по фазовому переменному. Из 1-однородности $\mathscr{A}$ вытекает инвариантность множества решений включения (1) относительно гомотетий: если $x(t)$ - решение включения $(1)$, то функция $c x(t), c \geqslant 0$, также есть решение этого включения. Экспоненциальная устойчивость нулевого решения в малом влечет справедливость оценки (4) для любого начального значения $x\left(t_{0}\right)$.

ЛЕмма 1. Пусть выполнено условие III и существуют такие положительные числа $T, K$ и $q<1$, что для любого решения $x$ включения (1) верны оценки

$$
\begin{gathered}
\max \left\{|x(t)|, t_{0} \leqslant t \leqslant t_{0}+T\right\} \leqslant K\left|x\left(t_{0}\right)\right|, \\
\min \left\{|x(t)|, t_{0} \leqslant t \leqslant t_{0}+T\right\} \leqslant q\left|x\left(t_{0}\right)\right| .
\end{gathered}
$$

Тогда для любого решения $x$ включения (1) справедлива оченка (4), в которой $B=K / q, \alpha=(1 / T) \ln (1 / q)$. 
ДокАЗАТЕЛЬСТво. Ввиду инвариантности множества решений включения (1) относительно гомотетий достаточно доказать оценку (4) в случае $\left|x\left(t_{0}\right)\right|=1$. Из (11) следует неравенство $|x(t)| \leqslant K, t_{0} \leqslant t \leqslant t_{0}+T$; оценка (12) влечет существование числа $t_{1} \in\left[t_{0}, t_{0}+T\right]$, для которого $\left|x\left(t_{1}\right)\right|=q$. Положим $c_{i}=q^{i}, i=1,2, \ldots$ Установим существование последовательности $t_{i}, i=1,2, \ldots$, обладающей свойствами

$$
|x(t)| \leqslant K q^{i-1}, \quad t_{i-1} \leqslant t \leqslant t_{i}, \quad\left|x\left(t_{i}\right)\right|=c_{i}, \quad 0<t_{i+1}-t_{i}<T .
$$

Существование $t_{1}$ уже установлено. Если известно $t_{i}$, то для построения $t_{i+1}$ введем функцию $u(t)=x(t) / c_{i}$. Она является решением включения (1). Очевидно, что $\left|u\left(t_{i}\right)\right|=1$. Заменяя в $(11),(12) t_{0}$ числом $t_{i}$, получаем, что $|u(t)| \leqslant K, t_{i} \leqslant t \leqslant t_{i}+T$ и $\left|u\left(t_{i+1}\right)\right|=q$ для некоторого $t_{i+1}$ из $\left[t_{i}, t_{i}+T\right]$. Число $t_{i+1}$ является искомьм; тем самым, последовательность $t_{i}$ определена при всех $i$.

Оценка (13) влечет неравенство $|x(t)| \leqslant K q^{i-1}$, если $t-t_{0} \geqslant(i-1) T, i=1,2, \ldots$. В частности, если $(i-1) T \leqslant t-t_{0} \leqslant i T$, то

$$
i \geqslant \frac{t-t_{0}}{T} \quad \text { и } \quad|x(t)| \leqslant \frac{K}{q} q^{i} \leqslant \frac{K}{q} q^{\left(t-t_{0}\right) / T}=B e^{-\alpha\left(t-t_{0}\right)}
$$

с $B=K / q, \alpha=(1 / T) \ln (1 / q)>0$. Лемма доказана.

Очевидно, что оценка (4) влечет оценки $(11),(12)$ с подходящими $T, K, q$. Таким образом, в случае 1 -однородного отображения $\mathscr{A}$ оценки $(11),(12)$ и (4) эквивалентны. Отметим, что при доказательстве леммы 1 условия I, II не использовались. В силу (7) неравенство (6) с $k_{1}=0$ влечет неравенство (11) с постоянной $K$, зависящей от $\left\|\gamma_{0} ; M_{1}\right\|$.

ЛЕмма 2. Пусть т-отображсение $\mathscr{A}$ удовлетворяет условию III и неравенству (6) с $k_{1}=0$. Если при некотором $T>0$

$$
\sup _{t_{0} \geqslant 0} \int_{t_{0}}^{t_{0}+T} \gamma_{0}(t) d t<0
$$

то нулевое решение включений (1) экспоненциально устойчиво.

ДокАЗАТЕЛьство. Утверждение вытекает из леммы 1 и оценки (7).

Наряду с дифференциальным включением (1) рассмотрим дифференциальное включение вида

$$
0 \in y^{\prime}+\mathscr{F}(t, y)
$$

где $\mathscr{F}$ - отображение класса $\mathbb{C}_{*}\left(\mathbb{R}_{+}, V\right)$. Если $0 \in \mathscr{F}(t, 0), t \in \mathbb{R}_{+}$, то включение $(14)$ имеет нулевое решение. Как и в п. 1 , определяются асимптотическая устойчивость и экспоненциальная устойчивость в малом нулевого решения включения (14). Если $m$-отображения $\mathscr{F}, \mathscr{A}: \mathbb{R}_{+} \times V \rightarrow V^{*}$ в определенном смысле близки, то устойчивость тривиального решения включения (14) может быть вьведена из теоремы 1. 
ТЕОрема 2. Пусть отображсение $\mathscr{A}$ удовлетворяет условиям теоремы 1. Пусть $\mathscr{F} \in \mathbb{C}_{*}\left(\mathbb{R}_{+}, V\right)$ и справедлива оченка

$$
\theta_{V^{*}}(\mathscr{F}(t, v), \mathscr{A}(t, v)) \leqslant \psi(|v|)\|v\|
$$

әде $t \geqslant 0, v \in V,|v| \leqslant R, R>0, \psi:[0, R] \rightarrow \mathbb{R}_{+}-$возрастающая функция, причем $\psi(+0)<\rho_{0}, \rho_{0}-$ положительная постоянная, фигурирующая в теореме 1 . Тогда нулевое решение включения (14) әкспоненциально устойчиво в малом.

ДокАЗАТЕЛЬСтво. Фиксируем $\rho$ из $\left(\psi(+0), \rho_{0}\right)$ и число $\eta>0$ так, что $\psi(s)<\rho$ при $s<\eta$. Поскольку $\rho<\rho_{0}$, то в силу теоремы 1 нулевое решение включения $(5)$ экспоненциально устойчиво в малом. Следовательно, найдется такое $\varepsilon>0$, что для решений $x$ включения (5) с $\left|x\left(t_{0}\right)\right|<\varepsilon$ верна оценка (4). Если $\delta>0$ и $B \delta<\min (\eta, \varepsilon)$, то для решений $x(t)$ включения $(5) \mathrm{c}\left|x\left(t_{0}\right)\right|<\delta$ справедливо неравенство

$$
|x(t)| \leqslant B\left|x\left(t_{0}\right)\right| \exp \left(-\alpha\left(t-t_{0}\right)\right)<B \delta<\eta, \quad t \geqslant t_{0}
$$

В этом случае $\psi(|x(t)|)<\rho$ и $\mathscr{F}(t, x(t)) \subset \mathscr{A}_{\rho}(t, x(t))$. Проведенное рассуждение означает, что для всех решений $x$ включения (14) с $\left|x\left(t_{0}\right)\right|<\delta$ имеет место оценка (4), т.е. нулевое решение включения (14) экспоненциально устойчиво в малом. Теорема доказана.

Теоремы 1, 2 можно рассматривать как многозначные варианты теорем об устойчивости по первому приближению. Условие $\psi(+0)<\rho_{0}$ в теореме 2 заведомо вьполняется, если $\psi(+0)=0$. Этот частньй случай удобен для проверки, так как не требует знания постоянной $\rho_{0}$ из теоремы 1.

3. Замечания и примеры. Предшествующие результаты могут быть распространены на случай, когда отображения $\mathscr{A}, \mathscr{A}_{\rho}, \mathscr{F}$ определены на цилиндре $\mathbb{R}_{+} \times\{v \in V$, $|v| \leqslant R\}$ с некоторым $R>0$. Это замечание существенно, например, при проверке условий I, II. Одностороннее условие Липшица, естественное для параболических включений, в ряде ситуаций оказьвается слишком жестким. Заменим условие I следующим менее обременительным предположением.

УСловиЕ IV. Порождаемый $\mathscr{A}$ оператор суперпозиции $A$ принадлежит классу $\alpha_{1}(Y(J), Z(J))$ для любого отрезка $J \subset \mathbb{R}_{+}$.

Введем еще одно определение. $M$-отображение $\mathscr{A}: \mathbb{R}_{+} \times V \rightarrow V^{*}$ назовем $\omega$-nериодuческим, $\omega>0$, если

$$
\mathscr{A}(t+\omega, v)=\mathscr{A}(t, v), \quad t \in \mathbb{R}_{+}, \quad v \in V .
$$

Из условия $\omega$-периодичности $\mathscr{A}$ следует инвариантность множества решений включения (1) относительно сдвига аргумента на $\omega$ : если $x(t)$ - решение включения $(1)$, то $x(t+\omega)$ - также есть решение включения (1).

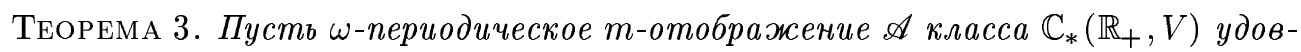
летворяет условиям II-IV и нулевое решение включения (1) асимптотически устойчиво. Тогда справедливо заключение теоремы 1. 
ДоКАЗАТЕЛЬСТво удобно разбить на ряд этапов. Обозначим через $\mathscr{R}$ множество решений $x$ включения $(1)$ с $|x(0)| \leqslant 1$. В силу асимптотической устойчивости нулевого решения включения (1) функции класса $\mathscr{R}$ равномерно ограничены в пространстве $H$.

Положим

$$
\eta_{n}=\sup \{|x(n \omega)|, x \in \mathscr{R}\} .
$$

На первом этапе докажем, что $\eta_{n} \rightarrow 0$ при $n \rightarrow \infty$. В предположении противного найдутся такое $\eta>0$ и такие функции $x_{n}$ из $\mathscr{R}$, что $\left|x_{n}(n \omega)\right| \geqslant \eta$ для бесконечного множества $\mathbb{N}_{0}$ индексов $n$. Фиксируем $\mu>0$ так, что для решений $y$ включения (1), удовлетворяющих неравенству $|y(0)|<\mu$, выполнялась оценка $|y(t)|<\eta$. Тогда для всех $x_{n}$, $n \subset \mathbb{N}_{0}$, справедливы соотношения

$$
\mu \leqslant\left|x_{n}(i \omega)\right| \quad \text { при } i=0,1, \ldots, n-1 .
$$

Действительно, если бы $\left|x_{n}\left(t^{*}\right)\right|<\mu$ при $t^{*}=i \omega$, то для решения $y(t)=x_{n}\left(t+t^{*}\right)$ включения (1) имели место неравенства $|y(0)|<\mu,\left|y\left(n \omega-t^{*}\right)\right|=\left|x_{n}(n \omega)\right| \geqslant \eta$, что противоречит выбору числа $\mu$.

Из оценки (8) следует ограниченность последовательности $x_{n}$ в пространстве $Y(J)$ для каждого отрезка $J=[0, b]$. Без ограничения общности можно считать, что последовательность $x_{n}, n \in \mathbb{N}_{0}$, сходится в $\sigma(Y(J), Z(J))$ к некоторой функции $x$. Согласно предложению $1 x$ есть решение включения (1) и $t x_{n} \rightarrow t x$ в $C(J, H)$. Для предельной функции $x$ верны оценки $|x(i \omega)| \geqslant \mu, i=1,2, \ldots$ Это противоречит асимптотической устойчивости нулевого решения включения (1). Итак, $\eta_{n} \rightarrow 0$, что завершает первьй этап доказательства.

Пусть

$$
K_{0}=\sup _{t_{0} \geqslant 0} \exp \left(\int_{t_{0}}^{t_{0}+\omega} \gamma_{0}(t) d t\right),
$$

$q_{0}>0$ и $q_{0} K_{0}<1$. В силу доказанного вьше найдется такое число $m$, что $\eta_{m}<q_{0}$. Фиксируем положительное число $\varepsilon$ так, что $\left(q_{0}+\varepsilon\right) K_{0}<1$. На втором этапе докажем существование такого $\rho_{0}>0$, что для решений $y$ включения $(5)$ с $|y(0)| \leqslant 1,0<\rho<\rho_{0}$ выполняется оценка $|y(m \omega)|<q_{0}+\varepsilon$.

В предположении противного можно считать, что существует последовательность $y_{n}$, $n=1,2, \ldots$, решений включения (5) при $\rho=\rho_{n}, \rho_{n}>0, \rho_{n} \rightarrow 0$, для которой $\left|y_{n}(0)\right| \leqslant 1$, но $\left|y_{n}(m \omega)\right| \geqslant q_{0}+\varepsilon$. Последовательность $y_{n}$ в силу оценки (9) ограничена в пространствах $C(J, H), Y(J)$, где $J=[0, m \omega]$. Без ограничения общности можно считать, что $y_{n} \rightarrow y$ в $\sigma(Y(J), Z(J))$. В силу предложения $1 y$ есть решение включения (1) и $t y_{n} \rightarrow t y$ в $C(J, H)$. В частности, $\left|y_{n}(m \omega)\right| \rightarrow|y(m \omega)|$, а так как $\left|y_{n}(m \omega)\right| \geqslant q_{0}+\varepsilon$, то $|y(m \omega)| \geqslant q_{0}+\varepsilon$. Из оценки (9) вытекает неравенство

$$
\left|y_{n}(t)\right| \leqslant \exp \Gamma_{0}(t), \quad \Gamma_{0}^{\prime}=\gamma_{0}, \quad \Gamma_{0}(0)=0,
$$

сохраняющееся и для предельной функции $y$. Следовательно, $y \in \mathscr{R}$; в таком случае $|y(m \omega)| \leqslant \eta_{m}<q_{0}$. Полученное противоречие завершает второй этап доказательства.

Фиксируем $\rho$ из $\left(0, \rho_{0}\right), \rho<k_{1}$. Если $x$ - решение включения $(5)$, то в силу выбора $\rho$ справедлива оценка $|x((n+m) \omega)| \leqslant\left(q_{0}+\varepsilon\right)|x(n \omega)|$ для любого натурального числа $n$. Пусть $t_{0} \geqslant 0, q=\left(q_{0}+\varepsilon\right) K_{0}<1$. Фиксируем натуральное число $n$ так, что 
$(n-1) \omega \leqslant t_{0} \leqslant n \omega$. Из оценки $(9)$ следует неравенство $|x(n \omega)| \leqslant K_{0}\left|x\left(t_{0}\right)\right|$. Объединяя установленные оценки, приходим к соотношению

$$
|x((n+m) \omega)| \leqslant\left(q_{0}+\varepsilon\right)|x(n \omega)| \leqslant K_{0}\left(q_{0}+\varepsilon\right)\left|x\left(t_{0}\right)\right| \leqslant q\left|x\left(t_{0}\right)\right| .
$$

Так как $(n+m) \omega-t_{0} \leqslant(m+1) \omega$, то (16) влечет $(12)$ с $T=(m+1) \omega$. Теперь экспоненциальная устойчивость нулевого решения включения (5) следует из леммы 1 . Теорема доказана.

Из теоремы 3 следует экспоненциальная устойчивость в малом тривиального решения включения (14), если $m$-отображение $\mathscr{F}$ близко к $m$-отображению $\mathscr{A}$; достаточно, например, чтобы имела место оценка (15). При этом $m$-отображение $\mathscr{F}$ может и не быть $\omega$-периодическим. Для конечномерных дифференциальных включений аналогичный теореме 3 результат установлен в [11]. В более ранних работах рассматривались автономные дифференциальные включения (см., например, [12] и приведенную там литературу).

Рассмотрим пример иллюстративного характера. Пусть $\Omega$ - ограниченная область в $\mathbb{R}^{n}, \stackrel{\circ}{W}_{2}^{1}(\Omega)$ - пространство Соболева $[13$, гл. 1$]$ функций $u: \Omega \rightarrow \mathbb{R}$, обращающихся в нуль на границе $\partial \Omega$ области $\Omega$. Норма в пространстве $V=\stackrel{\circ}{W}_{2}^{1}(\Omega)$ определена равенством $\|u\|=\left\|\nabla u ; L^{2}\left(\Omega, \mathbb{R}^{n}\right)\right\| ;$ здесь и далее

$$
\nabla u=\left(\frac{\partial u}{\partial x_{1}}, \ldots, \frac{\partial u}{\partial x_{n}}\right)_{V^{*}}
$$

- градиент функции $u$. Сопряженное к $V$ пространство состоит из функционалов $l$, допускающих представление

$$
l(u)=\int_{\Omega} f(x) \cdot \nabla u(x) d x,
$$

где $f \in L^{2}\left(\Omega, \mathbb{R}^{n}\right), \xi \cdot \xi^{*}$ - скалярное произведение векторов $\xi$ и $\xi^{*}$ из $\mathbb{R}^{n}$. Пространство $V$ компактно и плотно вложено в пространство $H=L^{2}(\Omega)$ с нормой $|u|=\left\|u ; L^{2}(\Omega)\right\|$. Пара пространств $V, H$ однозначно определяет соответствующие им пространства $Y$, $Z, W$ на отрезке $J \subset \mathbb{R}$. В частности, $Y=L^{2}\left(J, \stackrel{\circ}{W}_{2}^{1}(\Omega)\right) \cap L^{\infty}\left(J, L^{2}(\Omega)\right) ; Y$ можно интерпретировать как пространство функций на цилиндре $Q=\Omega \times J$. Совершенно аналогично, с каждой функцией $y: \mathbb{R}_{+} \rightarrow V$ можно связать функцию $y(x, t)$, определенную на цилиндре $Q_{+}=\Omega \times \mathbb{R}_{+}$. Ниже используется обозначение

$$
\nabla_{x} y=\left(\frac{\partial y}{\partial x_{1}}, \ldots, \frac{\partial y}{\partial x_{n}}\right)
$$

Через $\mathrm{Kv}\left(\mathbb{R}^{n}\right)$ обозначается совокупность непустых вьпуклых компактных подмножеств $\mathbb{R}^{n} ;|\xi|=\sqrt{\xi \cdot \xi}-$ евклидова норма элемента $\xi$ из $\mathbb{R}^{n}$.

Перейдем теперь к определению многозначных отображений на пространствах $V, Y$. Напомним, что $m$-отображение Г из топологического пространства $\mathscr{M}$ в $B$-пространство $E$ называют полунепрерывным сверху, если для любого открытого множества $G \subset t$ его мальй прообраз

$$
\Gamma_{+}^{-1}(G)=\{v \in \mathscr{M}, \Gamma(v) \subset G\}
$$


есть открытое подмножество $\mathscr{M}[2],[14]$. Обозначим через $\mathscr{U}\left(Q_{+}, \mathbb{R}^{n}\right)$ совокупность $m$-отображений $a: Q_{+} \times \mathbb{R}^{n} \rightarrow \mathrm{Kv}\left(\mathbb{R}^{n}\right)$, удовлетворяющих условиям

$\left.\mathrm{a}_{1}\right)$ при любом $\xi$ из $\mathbb{R}^{n}$ отображение $a(\cdot, \xi): Q_{+} \rightarrow \mathrm{Kv}\left(\mathbb{R}^{n}\right)$ имеет измеримое сечение, при всех $(x, t)$ из $Q_{+}$отображение $a(x, t, \cdot): \mathbb{R}^{n} \rightarrow \mathrm{Kv}\left(\mathbb{R}^{n}\right)$ полунепрерывно сверху;

a $) ~ a(x, t, c \xi)=c a(x, t, \xi),(x, t) \in Q_{+}, \xi \in \mathbb{R}^{n}, c>0$

$\left.\mathrm{a}_{3}\right)$ справедливы неравенства

$$
\left(\xi_{1}-\xi_{2}\right) \cdot\left(\xi_{1}^{*}-\xi_{2}^{*}\right) \geqslant 0, \quad \varkappa_{1}|\xi|^{2} \leqslant \xi \cdot \xi^{*}, \quad\left|\xi^{*}\right| \leqslant \varkappa_{2}|\xi|
$$

в которьх $\xi_{i} \in \mathbb{R}^{n}, \xi_{i}^{*} \in a\left(x, t, \xi_{i}\right), \varkappa_{i}$ - положительные постоянные $(i=1,2$; постоянные $\varkappa_{1}, \varkappa_{2}$ могут быть различными для разных отображений), $\xi \subset \mathbb{R}^{n}$, $\xi^{*} \in a(x, t, \xi)$.

Условие $\mathrm{a}_{1}$ ) можно рассматривать как многозначный аналог условия Каратеодори; в близкой форме оно использовалось в [15, с. 81]. Условие а2) означает 1-однородность отображения $a$ по $\xi$. Первое из неравенств в условии аз $)$ означает монотонность отображения $a(x, t, \cdot): \mathbb{R}^{n} \rightarrow \mathrm{Kv}\left(\mathbb{R}^{n}\right) ;$ два последних представляют собою оценки роста $a$. Условия $\left.\left.\mathrm{a}_{1}\right)-\mathrm{a}_{3}\right)$ вьполнены, например, если $m$-отображение $a$ имеет вид $a(x, t, \xi)=$ $\partial \Psi(\xi)$, где $\Psi: \mathbb{R}^{n} \rightarrow \mathbb{R}$ - вьпуклая 2-однородная функция, $\Psi(\xi)>0$ для $\xi \neq 0, \partial \Psi(\xi)-$ субдифференциал функции $\Psi$ в точке $\xi[2$, гл. 1 , п. 1]; в частности, можно положить $\Psi(\xi)=\|\xi\|^{2}\left(\|\cdot\|-\right.$ произвольная норма в $\left.\mathbb{R}^{n}\right)$.

Пусть $b: Q_{+} \rightarrow \mathbb{R}$ - функция класса $M_{1}\left(\mathbb{R}_{+}, L^{\infty}(\Omega)\right)$. Определим $m$-отображение $\mathscr{A}: \mathbb{R}_{+} \times V \rightarrow V^{*}$ равенством

$$
\mathscr{A}(t, v)=-\operatorname{div} a(\cdot, t, \nabla v)+b(\cdot, t) v
$$

Более точно, функционал $v^{*}$ на пространстве $V$ принадлежит $\mathscr{A}(t, v)$, если существует такое измеримое сечение $f$ отображения $\Gamma(x)=a(x, t, \nabla v(x))$, что

$$
\left(w, v^{*}\right)=\int_{\Omega}(f \cdot \nabla w+b \cdot(\cdot, t) w) d x \quad \forall w \in V
$$

В силу результатов работ [4], [6] отображение $\mathscr{A}$ принадлежит классу $\mathbb{C}_{*}\left(\mathbb{R}_{+}, V\right)$. Если $v_{j} \in V, v_{j}^{*} \in \mathscr{A}\left(y, v_{j}\right), j=1,2, t \in \mathbb{R}_{+}$, то измонотонности отображения $a(x, t, \cdot): \mathbb{R}^{n} \rightarrow$ $\mathrm{Kv}\left(\mathbb{R}^{n}\right)$ следует оценка

$$
\left(v_{1}-v_{2}, v_{1}^{*}-v_{2}^{*}\right) \geqslant \int_{\Omega} b(\cdot, t)\left|v_{1}-v_{2}\right|^{2} d x .
$$

Так как $b \in M_{1}\left(\mathbb{R}_{+}, L^{\infty}(\Omega)\right)$, то оценка (17) влечет выполнение условия I с функцией $\lambda(t)=-\left\|b(\cdot, t) ; L^{\infty}(\Omega)\right\|$ класса $M_{1}\left(\mathbb{R}_{+}\right)$. Для отображения $\mathscr{A}: \mathbb{R}_{+} \times V \rightarrow V^{*}$ выполнено условие II. Это без труда вытекает из неравенства $\xi \cdot \xi^{*} \geqslant \varkappa_{1}|\xi|^{2}\left(\xi \in \mathbb{R}^{n}\right.$, $\left.\xi^{*} \in a(x, t, \xi)\right)$ и включения $b \in M_{1}\left(\mathbb{R}_{+}, L^{\infty}(\Omega)\right)$. Наконец, условие III есть очевидное следствие 1-однородности отображения $a(x, t, \cdot)$.

Поскольку $\mathscr{A} \in \mathbb{C}_{*}\left(\mathbb{R}_{+}, V\right)$ и вьполнено условие I, то порождаемьй $\mathscr{A}$ оператор суперпозиции $A$ принадлежит классу $\alpha_{1}(Y(J), Z(J))$ для любого отрезка $J \subset \mathbb{R}_{+}$. B 
рассматриваемом случае решения задачи Коши $(1),(2)$ совпадают с обобщенными решениями начально-краевой задачи

$$
\begin{gathered}
0 \in \frac{\partial y}{\partial t}-\operatorname{div}\left(a\left(\cdot, t, \nabla_{x} y\right)\right)+b(\cdot t) y \\
\left.y(\cdot, t)\right|_{\partial \Omega}=0, \quad y\left(\cdot, t_{0}\right)=h .
\end{gathered}
$$

Для однозначного отображения $a$ данное определение решения совпадает с принятым в теории параболических уравнений (см., например, [1], [8], [13]). Задача Коши (18), (19) имеет единственное решение $y$ при любых $t_{0} \geqslant 0, h \in L^{2}(\Omega)$.

Для исследования устойчивости нулевого решения этой задачи можно использовать предшествуюшие результаты. Приведем пример применения леммы 2. В изучаемом случае отображение $\mathscr{A}$ удовлетворяет условию III. Справедливо вытекающее из условия а 3 ) неравенство

$$
\left(v, v^{*}\right) \geqslant \int_{\Omega}\left(\varkappa_{1}|\nabla v|^{2}+b(\cdot, t) v^{2}\right) d x,
$$

где $v \in V, v^{*} \in \mathscr{A}(t, v), t \geqslant 0$. Обозначим через $\lambda_{1}(t)$ первое собственное значение задачи

$$
-\varkappa_{1} \Delta u+b(\cdot, t) u=\lambda u,\left.\quad u\right|_{\partial \Omega}=0 .
$$

Так как $b \in M_{1}\left(\mathbb{R}_{+}, L^{\infty}(\Omega)\right)$, то $\lambda_{1} \in M_{1}\left(\mathbb{R}_{+}\right)$. Из определения функции $\lambda_{1}(t)$ вытекает оценка

$$
\int_{\Omega}\left(\varkappa_{1}|\nabla v|^{2}+b(\cdot, t) v^{2}\right) d x \geqslant \lambda_{1}(t) \int_{\Omega} v^{2} d x, \quad v \in V .
$$

Неравенства (20), (21) означают, что отображение $\mathscr{A}$ удовлетворяет неравенству (6) с $k_{1}=0, \gamma_{0}(t)=-\lambda_{1}(t)$. В силу леммы 2 нулевое решение включения (18) экспоненциально устойчиво, если при некотором $T>0$

$$
\inf _{t_{0} \geqslant 0} \int_{t_{0}}^{t_{0}+T} \lambda_{1}(t) d t>0
$$

Наряду с включением (18) рассмотрим его возмущение - включение

$$
0 \in \frac{\partial y}{\partial t}-\operatorname{div}\left(a\left(\cdot, t, \nabla_{x} y\right)\right)+b(\cdot, t) y+g(\cdot, t, y)
$$

где функция $g: Q_{+} \times \mathbb{R} \rightarrow \mathbb{R}$ удовлетворяет условию Каратеодори $[16$, гл. 5] и неравенству $|g(x, t, \eta)| \leqslant$ const $|\eta|^{r}, 1 \leqslant r<1+4 / n$. Определяемьй равенством $\mathscr{G}(t) v=g(\cdot, t, v)$ оператор суперпозиции действует и непрерьвен из пространства $V$ в сопряженное к нему пространство $V^{*}$. Справедлива оценка

$$
\left\|\mathscr{G}(t) v ; V^{*}\right\| \leqslant \varkappa\|v\||v|^{r-1}, \quad v \in V
$$

с некоторой постоянной $\varkappa>0$. Для доказательства (23) достаточно воспользоваться мультипликативным неравенством [13, с. 79]

$$
\left\|v ; L^{r p}\right\| \leqslant \text { const }\|v\|^{1 / r}|v|^{1-1 / r}
$$


где $p \in(1, \infty)$ и таково, что $L^{p}(\Omega) \rightarrow V^{*} ;$ возможность подобного выбора $p$ вытекает из теорем вложения и неравенства $1<r<1+4 / n$.

Положим $\mathscr{F}(t, v)=\mathscr{A}(t, v)+\mathscr{G}(t, v)$. Оценка $(23)$ влечет неравенство $(17)$ с $\psi(t)=$ $\varkappa|t|^{r-1}$. В данном случае $\psi(+0)=0$, поэтому применима теорема 2 , в соответствии с которой нулевое решение включения (22) экспоненциально устойчиво в малом, если аналогичньм свойством обладает нулевое решение включения (18).

Развитая выше схема применима, разумеется, и к более общим, чем (22), параболическим включениям. Дополнительная библиография, относяшаяся к затронутым вьше вопросам, может быть найдена в [8]-[16].

\section{СПИСОК ЦИТИРОВАННОЙ ЛИТЕРАТУРЫ}

[1] Гаевский Г., Грёгер К., Захариас К. Нелинейные операторные уравнения и операторные дифференциальные уравнения. М.: Мир, 1978.

[2] Обен ЖК. П., Экланд И. Прикладной нелинейный анализ. М.: Мир, І988.

[3] Климов В. С. К задаче о периодических решениях операторных дифференциальных включений // Изв. АН СССР. Сер. матем. 1989. Т. 53. № 2. С. 309-327.

[4] Климов В. С. Эволюционные параболические неравенства с многозначными операторами // Матем. сб. 1993. Т. 184. № 8. С. 37-54.

[5] Похожаев С.И. О разрешимости нелинейных уравнений с нечетными операторами // Функцион. анализ и его прилож. 1967. Т. 1. № 3. С. 48- 55.

[6] Климов В.С. Об операторе сдвига по траекториям параболических включений // Дифоференц. уравнения. 1995. Т. 31. №10. С. 1716-1721.

[7] Климов В. С. Ограниченные решения дифференциальных включений с однородной главной частью // Изв. РАН. Сер. матем. 2000. Т. 64. № 4. С. 109-130.

[8] Дубинский Ю. А. Нелинейные эллиптические и параболические уравнения // Современные проблемы математики. 1976. Т. 9. С. 5-130.

[9] Барбашин Е. А. Введение в теорию устойчивости. М.: Наука, 1967.

[10] Далецкий Ю. Л., Крейн М. Г. Устойчивость решений дифференциальных уравнений в банаховом пространстве. М.: Наука, 1970.

[11] Морозов М.В.О свойствах периодических дифференциальных включений // Дифференц. уравнения. 2000. Т. 36. № 5. С. 612-617.

[12] Филиппов А. Ф. Дифференциальные уравнения с разрьвной правой частью. М.: Наука, 1985.

[13] Ладыженская О. А., Солонников В. А., Уральцева Н. Н. Линейные и квазилинейные уравнения параболического типа. М.: Наука, 1967.

[14] Борисович Ю.Г., Гельман Б. Д., Мышкис А. Д., Обуховский В. В. Топологические методы в теории неподвижных точек многозначных отображений // УМН. 1960. Т. 35. № 1. C. 59-126.

[15] Толстоногов А. А. Дифференциальные включения в банаховом пространстве. Новосибирск: Наука, 1986.

[16] Красносельский М. А., Забрейко П. П., Пустыльник Е. И., Соболевский П. Е. Интегральные операторы в пространствах суммируемых функций. М.: Наука, 1966. 